\title{
Investigação da Influência do Processamento na Dinâmica Molecular de Nanocompósitos de Policarbonato e Argila Organofílica Obtidos via Intercalação por Fusão
}

\author{
Elton J. da R. Rodrigues, Suéllen A. M. Nascimento \\ Instituto de Macromoléculas Professora Eloisa Mano, UFRJ \\ Departamento de Química, FTESM \\ Maria I. B. Tavares \\ Instituto de Macromoléculas Professora Eloisa Mano, UFRJ \\ Pedro P. Merat \\ Instituto de Macromoléculas Professora Eloisa Mano, UFRJ \\ Instituto Federal de Educação, Ciência e Tecnologia Rio de Janeiro
}

\begin{abstract}
Resumo: Nanocompósitos baseados em policarbonato e argila organofílica foram obtidos pelo método de intercalação por fusão em câmara de mistura sob diferentes valores de torque e temperatura. A influência desses parâmetros na qualidade da dispersão da argila na matriz polimérica foi investigada por ressonância magnética nuclear (RMN) de baixo campo, empregando o tempo de relaxação longitudinal, $\mathrm{T}_{1} \mathrm{H}$, e por difração de raios $\mathrm{X}$. As diferenças nos tempos de relaxação longitudinal e nos padrões de difração de raios X foram correlacionadas com a formação de domínios com mobilidades distintas. O nanomaterial formado apresentou morfologia intercalada com afastamento crescente das camadas de argila, conforme as condições de processamento se tornavam mais severas. A RMN ${ }^{1} \mathrm{H}$ mostrou que tanto o cisalhamento quanto a temperatura afetaram a mobilidade das cadeias poliméricas, explicitando a homogeneidade crescente da distribuição dos domínios de relaxação, de acordo com o aumento do torque e da temperatura.
\end{abstract}

Palavras-chave: Nanocompósito, intercalação por fusão, RMN.

\section{On the Influence of Processing Parameters on the Molecular Dynamics of Melt Intercalated Polycarbonate-clay Nanocomposites}

\begin{abstract}
Polycarbonate-organofilic clay nanocomposites were obtained via the melt intercalation method in the blending chamber of a torque rheometer, under distinct values of shear and temperature. The influence of those processing parameters on the clay dispersion in the polymer matrix was probed by low-field nuclear magnetic resonance (NMR), using the $\mathrm{T}_{1} \mathrm{H}$ measurement technique and by wide angle X-ray scattering (WAXS). The differences in longitudinal relaxation times and in the X-ray reflection patterns were correlated with the formation of relaxation domains with particular molecular mobility, as progressively larger intercalated morphologies were found in the nanocomposites due to the increase of shear stress and temperature. ${ }^{1} \mathrm{H}$ NMR has shown that both shearing and temperature have played a fundamental part on the polymer chains mobility, which explains the increase in the domain's homogeneity following the increment of those processing parameters.
\end{abstract}

Keywords: Nanocomposite, melt intercalation, NMR.

\section{Introdução}

Nanocompósitos poliméricos vêm ganhando atenção crescente no âmbito da pesquisa mundial nas últimas décadas ${ }^{[1,2]}$. Os nanomateriais híbridos, formados pela matriz polimérica e uma carga geralmente mineral que apresenta ao menos uma dimensão em escala de ordem nanométrica $\left(10^{-9} \mathrm{~m}\right)$, são obtidos tradicionalmente por meio de quatro rotas: método sol-gel, polimerização in situ, intercalação por solução (solution casting) e intercalação no estado fundido ${ }^{[1,3-5]}$.

A intercalação no estado fundido é o método mais rápido dentre os três apresentados para obtenção de nanocompósitos. Além de não utilizar solventes potencialmente nocivos ao meio ambiente e à saúde, tem a vantagem dos nanomateriais assim obtidos poderem ser moldados diretamente em formas específicas para sua aplicação, sem necessidade de processamento adicional ${ }^{[1]}$.

Dentre os vários tipos de nanocompósitos existentes, os formados por polímeros e silicatos lamelares se destacam por suas propriedades mecânicas, sua boa resistência ao calor e à oxidação, transparência óptica e biodegradabilidade, além de apresentarem baixa permeabilidade aos $\operatorname{gases}^{[6-11]}$.

A argila mais empregada em pesquisa na área de nanocompósitos baseados em polímero-silicatos é a bentonita, pertencente ao grupo das esmectitas. Estas, cujo argilomineral predominante é a montmorilonita, caracterizam-se por possuir estruturas lamelares do tipo 2:1, com duas folhas tetraédricas de sílica e uma folha octaédrica de alumina ${ }^{[1,12]}$. Estas argilas são modificadas quimicamente de maneira a tornarem-se organofílicas. Este processo envolve a troca dos cátions livres na galeria entre as camadas da argila por sais graxos de amônio quaternário, que são os mais comumente empregados dentre os surfactantes disponíveis ${ }^{[1,2]}$.

As características térmicas, mecânicas, ópticas, elétricas e de barreira apresentadas por nanocompósitos de polímero-argila

Autor para correspondência: Maria I. B. Tavares, Instituto de Macromoléculas Professora Eloisa Mano, Universidade Federal do Rio de Janeiro - UFRJ, 
organofílica advêm da íntima interface polímero-carga proporcionada pela forte interação em escala nanométrica ${ }^{[8,13-17]}$. $\mathrm{O}$ emprego de quantidades reduzidas dessas nanocargas sugere preservação das propriedades da matriz polimérica aliada ao ganho em certas características do nanomaterial sobre o polímero puro. A extensão do ganho nessas propriedades é função da qualidade de dispersão, do tipo de nanocarga e da morfologia do nanocompósito obtido $^{[18]}$

A análise da dinâmica molecular do híbrido obtido torna-se fundamental na elucidação da qualidade da interação polímero-carga. A melhor compreensão da homogeneidade do material pode ser conseguida por meio da medida dos tempos de relaxação do núcleo do ${ }^{1} \mathrm{H}$ no estado sólido, que vem se mostrando uma técnica poderosa na caracterização de materiais poliméricos. A difração de raios $\mathrm{X}$ pode ser empregada como técnica auxiliar, por ser um método tradicional de análise, auxiliando na corroboração dos resultados explicitados pela $\mathrm{RMN}{ }^{1} \mathrm{H}^{[19]}$.

Neste trabalho, investigou-se a qualidade da dispersão da argila organofílica na matriz de policarbonato em função da variação do torque e da temperatura da câmara de mistura. Para tanto, combinou-se os dados da difratometria de raios $\mathrm{X}$ com aqueles obtidos pela técnica de relaxometria por RMN de baixo campo. A relaxação longitudinal do núcleo de hidrogênio foi empregada na análise dos domínios formados nos materiais estudados.

\section{Experimental}

\section{Materiais recebidos}

O polímero usado neste estudo foi o Policarbonato (PC) fornecido pela Policarbonato S. A. Indústria e Comércio - Camaçarí/BA, com massa molecular ponderal média $(\mathrm{Mw})$ de 55.600, conforme informado pelo fabricante.

A argila montmorilonita modificada organicamente (OMMT) com sal de estearilamônio foi fornecida pela Bentec Aditivos Reológicos.

Ambos os materiais foram usados como recebidos, sem sofrer modificações adicionais.

\section{Obtenção dos nanocompósitos}

Pesou-se quantidade suficiente de polímero e argila para obter seis amostras, sendo três de polímero puro e três de uma mistura polímero e argila com $1 \%(\mathrm{~m} / \mathrm{m})$ da última.

O material foi processado na câmara de mistura de um reômetro de torque Haake Rheocord 9000, com misturadores do tipo roller em três bateladas, cada uma com temperatura e torque crescentes $\left(240^{\circ} \mathrm{C}\right.$ e $30 \mathrm{rpm}, 270{ }^{\circ} \mathrm{C}$ e $60 \mathrm{rpm}, 300^{\circ} \mathrm{C}$ e $90 \mathrm{rpm}$ ) por 10 minutos.

Os nanocompósitos obtidos, doravante chamados de PC1, PC2, PC3 e PCOMMT1, PCOMMT2, PCOMMT3, nomeados em ordem crescente de torque e temperatura, foram retirados da câmara e deixados em repouso até retornar à temperatura ambiente. Posteriormente, foram prensados a $180{ }^{\circ} \mathrm{C}$ com $7 \mathrm{t}$ de pressão até formarem filmes.

\section{Caracterizações dos nanocompósitos}

Os materiais iniciais, como o polímero e a argila, também foram analisados por difração de raios $\mathrm{X}$ e relaxação nuclear à temperatura ambiente para acompanhar as eventuais modificações estruturais ocorridas no processamento.

Os filmes foram submetidos à análise de difração de raios $\mathrm{X}$ em um difratômetro Rigaku Miniflex com emissão de radiação de $\mathrm{CuK} \alpha(\lambda=1,5418 \AA)$ à temperatura ambiente, em $40 \mathrm{kV}$ e $30 \mathrm{~mA}$.
Os padrões de difração foram coletados a uma taxa de varredura de $2^{\circ}<2 \theta<70^{\circ}$, por 1 segundo, passo de $0,05^{\circ}$.

A análise da relaxação nuclear do próton foi realizada em um aparelho Resonance Instruments Maran Ultra 23, à temperatura de $27^{\circ} \mathrm{C}$ empregando os parâmetros operacionais, listados na Tabela 1 .

Os tempos de relaxação spin-rede do núcleo de $\mathrm{T}_{1} \mathrm{H}$, determinados pela técnica de inversão-recuperação, foram ajustados por meio do programa WinFit. As curvas de distribuição para os domínios com diferentes mobilidades moleculares foram ajustadas utilizando múltiplas exponenciais por meio do programa WinDXP.

\section{Resultados e Discussões}

\section{Difração de raios $X$}

A Figura 1 mostra o padrão de difração de raios $\mathrm{X}$ do polímero depois de cada condição de processamento.

A análise dos padrões de difração do polímero não indicou alterações perceptíveis, derivadas dos efeitos e/ou influência dos parâmetros de processamento na estrutura cristalina do polímero. A Figura 2 mostra o padrão de difração da OMMT:

Na Figura 2, nota-se o pico $2 \theta=3,4^{\circ}$, que corresponde ao plano cristalino $d_{(001)}$ da argila. De acordo com a Lei de Bragg $(\mathrm{n} \lambda=2 \mathrm{~d}$. $\operatorname{sen} \theta)$, este pico possui uma distância interplanar de $2,6 \mathrm{~nm}$. A tendência é que, em função da formação do nanocompósito, haja um deslocamento desse pico para ângulos menores, provocando um aumento desse espaçamento entre as lamelas da argila. A desordem causada na organização cristalina da argila, devido à entrada das cadeias poliméricas em sua estrutura, aumenta ainda mais este espaçamento, podendo levar à esfoliação das lamelas (Figura 3$)^{[1,2]}$.

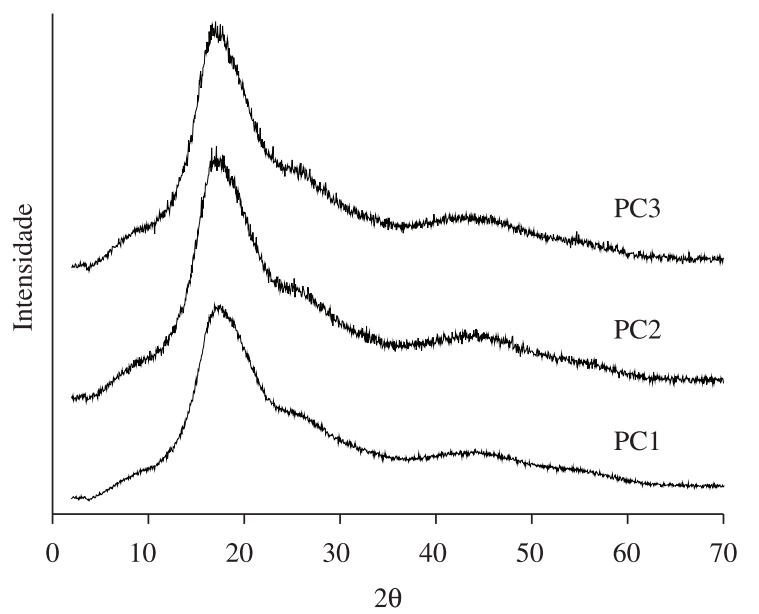

Figura 1. Difratogramas de raios $\mathrm{X}$ do $\mathrm{PC}$ em diferentes processamentos.

Tabela 1. Parâmetros operacionais de RMN.

\begin{tabular}{lc}
\hline \multicolumn{1}{c}{ Parâmetro } & Valores \\
\hline Freqüência observação ${ }^{1} \mathrm{H}(\mathrm{MHz})$ & 23 \\
Tempo de aquisição (segundos) & 0,024 \\
Janela espectral $(\mathrm{Hz})$ & 4999999,9 \\
Largura de pulso $(\pi / 2, \mu \mathrm{s})$ & 4,2 \\
Largura de pulso $(\pi, \mu \mathrm{s})$ & 8,4 \\
Intervalo entre os pulsos (segundos) & 5 \\
Faixa de $\tau(\mu \mathrm{s})$ & $100-5.000 .000$ \\
$\mathrm{~N}^{\circ}$ de acumulos & 4 \\
Números de pontos & 40 \\
\hline
\end{tabular}


O deslocamento do pico basal de difração da argila para ângulos menores indicou a formação de um material, com certo grau de intercalação das cadeias poliméricas, entre as lamelas do argilomineral e seu progressivo afastamento. A distância interlamelar verificada após a obtenção do nanocompósito foi de 3,0 nm para PCOMMT1, 3,2 nm para PCOMMT2 e 3,3 nm para PCOMMT 3. Além disso, neste último, a redução da intensidade da

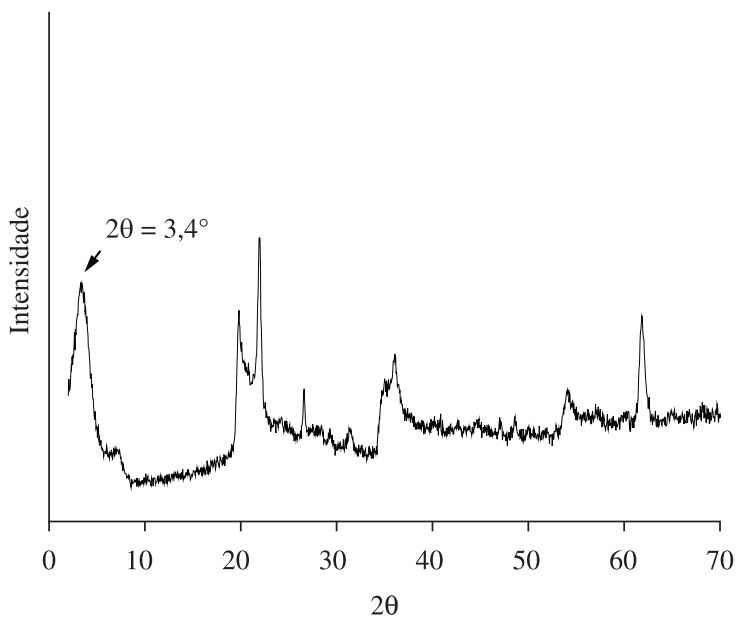

Figura 2. Difratograma da argila organofílica.

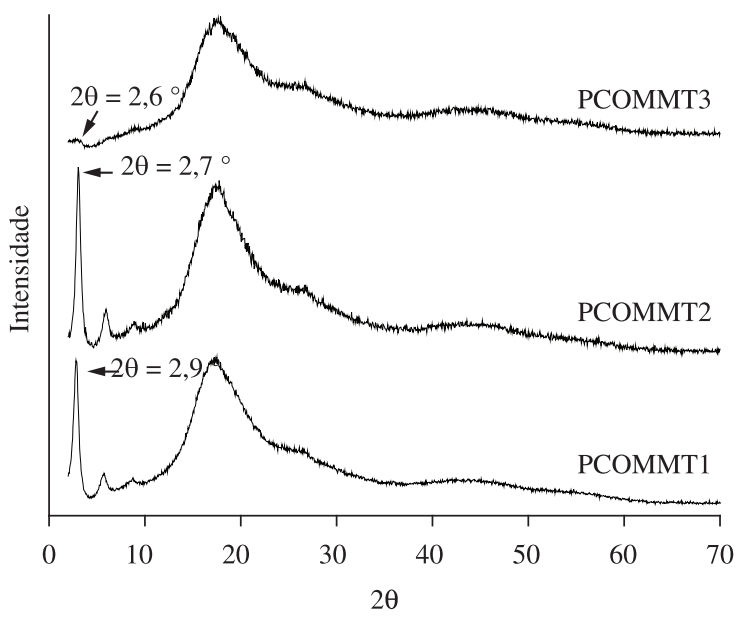

Figura 3. Difratogramas dos nanocompósitos de PC e OMMT.

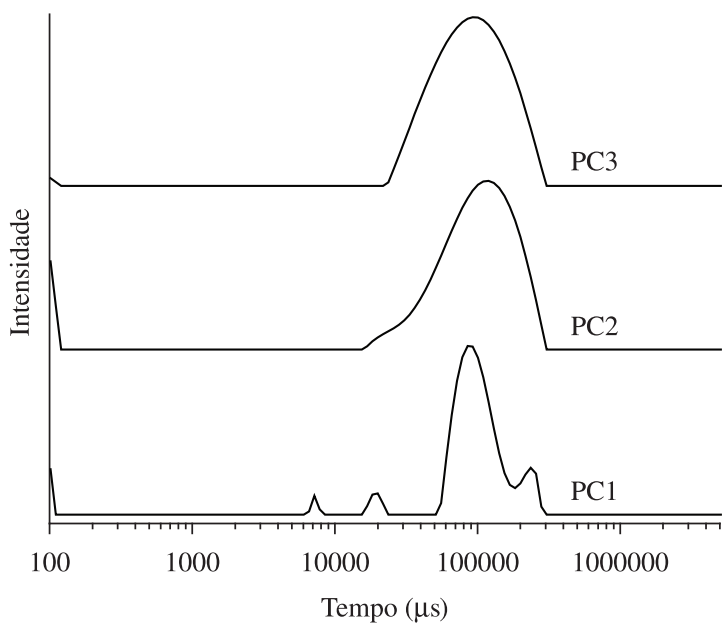

Figura 4. Curvas de distribuição de domínios de $\mathrm{T}_{1} \mathrm{H}$ do polímero. reflexão do plano basal da argila indica a redução na coerência de empilhamento das camadas ${ }^{[1]}$.

\section{Ressonância magnética nuclear de baixo campo}

Com o objetivo de obter informações mais detalhadas sobre a dinâmica molecular dos nanocompósitos de PC e OMMT, foi determinado o tempo de relaxação spin-rede do núcleo de hidrogênio $\left(\mathrm{T}_{1} \mathrm{H}\right)$, por RMN em baixo campo no estado sólido, utilizando a técnica inversão-recuperação.

\section{Análise de RMN do polímero}

A curva de domínio (Figura 4) mostra as mudanças ocorridas na dinâmica molecular do polímero por efeito do processamento.

As formas assumidas pelas distribuições dos domínios de relaxação de ${ }^{1} \mathrm{H}$ mostraram que tanto o cisalhamento quanto a temperatura afetaram a mobilidade das cadeias poliméricas. A homogeneidade crescente na distribuição dos domínios de relaxação variou de acordo com o aumento da temperatura e do torque.

\section{Análises de RMN da argila organofílica}

A Figura 5 exibe a curva de distribuição de domínios de $\mathrm{T}_{1} \mathrm{H}$ da argila organofílica, antes de sua incorporação na matriz polimérica:

A alta mobilidade apresentada pelas cadeias hidrocarbônicas do agente surfactante deve-se primariamente ao teor $\mathrm{de} \mathrm{Fe}^{3+}$ presente por conta de eventuais substituições isomórficas na estrutura octaédrica da argila ${ }^{[1]}$. O paramagnetismo do íon metálico causa decréscimo líquido dos tempos de relaxação do núcleo do hidrogênio ${ }^{[19]}$.

\section{Análise de RMN dos nanocompósitos}

A curva de domínio (Figura 6) mostra homogeneidade crescente no material, acompanhando o aumento de temperatura e torque.

A homogeneidade e qualidade da dispersão da argila organofílica no nanocompósito também foi verificada determinando-se os tempos de relaxação spin-rede dos materiais, conforme pode ser visto na Tabela 2 .

A qualidade da dispersão da argila organofílica é inversamente proporcional ao tempo de relaxação dos domínios formados no nanocompósito. Quanto mais dispersa a argila estiver no meio (predominância de característica esfoliada), mais extenso e íntimo será o contato das cadeias poliméricas com as estruturas lamelares contendo íons paramagnéticos. Desta forma, o efeito de redução no tempo de relaxação dessas cadeias tornou-se mais evidente ${ }^{[19]}$. Os dados da Tabela 2 confirmaram essa tendência nos casos de PCOMMT1 e PCOMMT2.

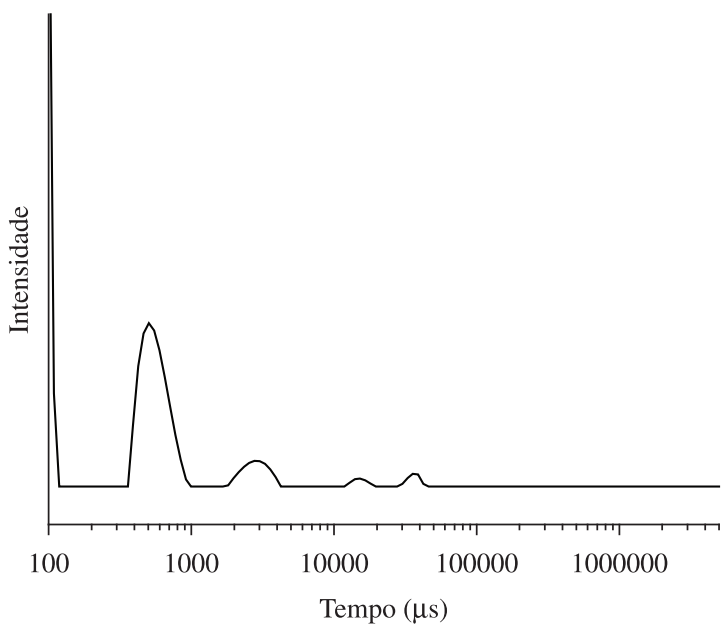

Figura 5. Curvas de distribuição de domínios de $\mathrm{T}_{1} \mathrm{H}$ da argila organofílica. 


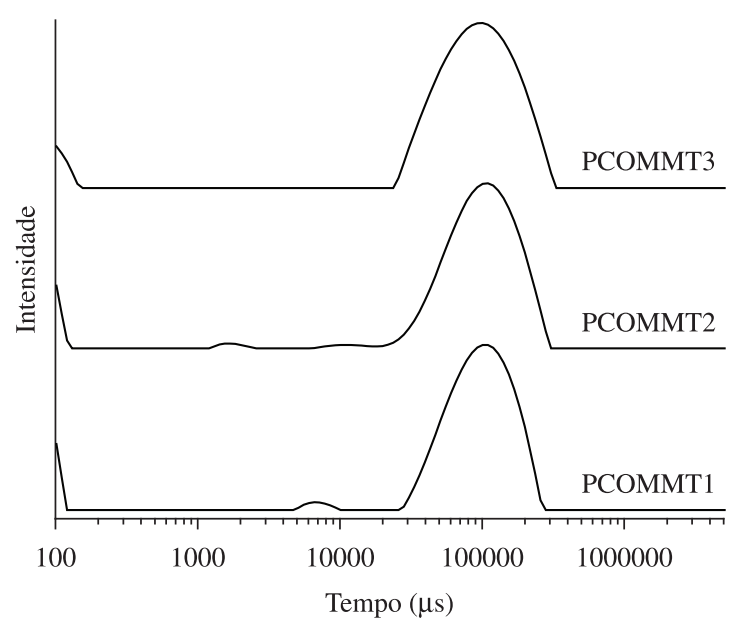

Figura 6. Curvas de distribuição de domínios de $\mathrm{T}_{1} \mathrm{H}$ dos nanocompósitos.

Tabela 2. Valores de tempos de relaxação spin-rede para PC e PC/OMMT.

\begin{tabular}{|c|c|c|c|c|c|}
\hline Material & $\begin{array}{l}\mathrm{T}_{1} \mathbf{H} \\
(\mathrm{ms})\end{array}$ & $\begin{array}{c}\text { Domínio de } \\
\mathrm{T}_{1} \mathrm{H}(\%)\end{array}$ & Material & $\begin{array}{l}\mathrm{T}_{1} \mathbf{H} \\
(\mathbf{m s})\end{array}$ & $\begin{array}{c}\text { Domínio de } \\
\text { T }_{1} \text { H }(\%)\end{array}$ \\
\hline \multirow{2}{*}{$\mathrm{PC} 1$} & 42 & 27 & \multirow{2}{*}{ PCOMMT1 } & 28 & 17 \\
\hline & 125 & 73 & & 114 & 83 \\
\hline $\mathrm{PC} 2$ & 95 & & PCOMMT2 & 93 & \\
\hline $\mathrm{PC} 3$ & 87 & & PCOMMT3 & 88 & \\
\hline
\end{tabular}

A morfologia intercalada de nanocompósito apresenta um fator extra na modificação do tempo de relaxação spin-rede das cadeias poliméricas: o efeito corset. Este fenômeno consiste no confinamento das cadeias do polímero em nanoestruturas formadas por sua entrada nos espaços interlamelares da argila. $\mathrm{O}$ efeito corset aumenta o tempo de relaxação líquido dessas cadeias, já que estas perdem parte de sua mobilidade molecular, muito embora ainda haja redução naquele tempo por contribuição da fração de cadeias que estão próximas da estrutura da argila ${ }^{[19]}$. Verificou-se a ocorrência desse efeito, de maneira mais pronunciada, no caso de PCOMMT3. $\mathrm{O}$ cisalhamento intenso e a alta temperatura, empregados na obtenção deste nanocompósito, podem ter colaborado para facilitar a entrada de segmentos de cadeias poliméricas nas galerias da argila, aumentando seu confinamento e diminuindo levemente sua mobilidade molecular.

\section{Conclusões}

Nanocompósitos de PC e argila organofílica com morfologia mista foram obtidos por intercalação no estado fundido.

A partir da comparação entre os resultados de difração de raios $\mathrm{X}$ em conjunto com os resultados de RMN de baixo campo magnético foi possível obter maiores informações a respeito da dinâmica molecular, qualidade de dispersão da nanocarga na matriz polimérica e homogeneidade do nanomaterial.

A RMN ${ }^{1} \mathrm{H}$ mostrou-se útil e, ainda, complementar às análises de difração de raios $X$, propiciando a elucidação da dinâmica molecular tanto do polímero quanto dos nanocompósitos obtidos.

\section{Agradecimentos}

Os autores agradecem aos órgãos governamentais de fomento à pesquisa científica pelas bolsas de estudo dos autores.

\section{Referências Bibliográficas}

1. Pavlidou, S. \& Papaspyrides, C.D. - Prog. Polym. Sci., 33, p.1119 (2008). http://dx.doi.org/10.1016/j.progpolymsci.2008.07.008

2. Ray, S. S. \& Okamoto, M. - Prog. Polym. Sci., 28, p.1539 (2003). http://dx.doi.org/10.1016/j.progpolymsci.2003.08.002

3. Liu, T.; Burger, C. \& Chu, B. - Prog. Polym. Sci., 28, p.5 (2003). http:// dx.doi.org/10.1016/S0079-6700(02)00077-1

4. Giannelis, E. P. - Adv. Mater., 8, p.29 (1996). http://dx.doi.org/10.1002/ adma. 19960080104

5. Le Baron, P. C.; Wang, Z. \& Pinnavaia, T. J. - Appl. Clay Sci., 15, p.11 (1999). http://dx.doi.org/10.1016/S0169-1317(99)00017-4

6. Schmidt, D.; Shah, D. \& Giannelis E.P. - Curr. Opin. Solid St. M., 6, p.205 (2002). http://dx.doi.org/10.1016/S1359-0286(02)00049-9

7. Shen, Z.; Simon, G. P. \& Chrng, Y. - Polymer, 43, p.4251 (2002). http:// dx.doi.org/10.1016/S0032-3861(02)00230-6

8. Tang, Y.; Hu, Y.; Song, L.; Zong, R.; Gui, Z.; Chen, Z. \& Fan, W. - Polym. Degrad. Stabil., 82, p.127 (2003). http://dx.doi.org/10.1016/S01413910(03)00173-3

9. Kojima, Y.; Usuki, A.; Kawasumi, M.; Okada, A.; Kurauchi, T. \& Kamigaito, O. - J. Polym. Sci., 31, p.1755 (1993). http://dx.doi. org/10.1002/pola.1993.080310714

10. Vu, T. Y.; Mark, J. E.; Pham, L. H. \& Engelhartd, M. - J. Appl. Polym. Sci., 82, p.1391 (2001). http://dx.doi.org/10.1002/app.1976

11. Joly, S.; Gaunaud, G.; Ollitrault, R.; Bokobza, L. \& Mark, J. E. - Chem. Mater., 14, p.4202 (2002). http://dx.doi.org/10.1021/cm020093e

12. Arroyo, M.; López-Manchado, M. A. \& Herrero, B. - Polymer, 44, p.2447 (2003). http://dx.doi.org/10.1016/S0032-3861(03)00090-9

13. Gilman, J. W.; Jackson, C. L.; Morgan, A. B.; Harris, R. J.; Manias, E.; Giannelis, E. P.; Wuthenow, M.; Hilton, D. \& Phillips, S. H. - Chem. Mater., 12, p.1866 (2000). http://dx.doi.org/10.1021/cm0001760

14. Alexandre, M. \& Dubois, P. - Mater. Sci. Eng., 28, p.1 (2000).

15. Castelvetro, V. \& Vita, C.D. - Adv. Colloid Interfac., 108-109, p.167 (2004). http://dx.doi.org/10.1016/j.cis.2003.10.017

16. Matejka, L.; Dusek, K.; Plestil, J.; Kriz, J. \& Lednicky, F. - Polymer, 40, p.171 (1999). http://dx.doi.org/10.1016/S0032-3861(98)00214-6

17. Ji, X.L; Jing, J.K.; Jiang, W. \& Jiang, B.Z. - Polym. Eng. Sci., 42, p.1023 (2002). http://dx.doi.org/10.1002/pen.11007

18. Esteves, A. C. C.; Timmons, A. B. \& Trindade, T. - Quim. Nova, 27, p.798 (2004). http://dx.doi.org/10.1590/S0100-40422004000500020

19. Tavares, M.I.B.; Nogueira, R. F.; Gil, R. A. S. S.; Preto, M.; Silva, E. O.; Silva, M. B. R. \& Miguez, E. - Polym. Test., 26, p.1100 (2007). http://dx.doi.org/10.1016/j.polymertesting.2007.07.012

Enviado: 09/05/11

Reenviado: $26 / 10 / 11$

Aceito: $21 / 12 / 11$ 